\title{
Ideas Esenciales para Escribir un Artículo de Revisión en Psicología de la Educación
}

\section{Essential Tips for Writing Literature Review Papers in Educational Psychology}

\author{
Carlos E. Zerpa ${ }^{1}$ \\ Universidad Central de Venezuela, Venezuela \\ Jorge J. Ramírez L. \\ Universidad Simón Bolívar, Venezuela
}

(Recepción: Julio 2008 - Aceptación: Octubre 2008)

\begin{abstract}
Resumen
Este artículo presenta de forma sucinta un conjunto de ideas básicas para organizar y escribir un artículo de revisión; se siguen las prescripciones derivadas del Manual de Publicaciones de la APA e indicaciones precisas de un editor de una afamada publicación (Daryl Bem) y de un académico especialista en metodología de la investigación (José Galván), así mismo, se presentan algunos de los errores comunes que se suelen cometer al momento de escribir un artículo; por último, se propone una integración de las fases de composición de texto con algunas estrategias de auto-regulación como una posible guía para orientar la actividad estratégica de los autores al abordar el proceso de escritura de un artículo de revisión en Educación y/o Psicología.
\end{abstract}

Palabras Clave: Artículos de revisión, APA, metodología de la investigación.

\begin{abstract}
This paper briefly presents some basic ideas on how to organize and write review papers for scientific journals. Prescriptions are derived from Publication Manual-APA, as well as precise indications from a famous editor of a prestigious publication (Daryl Bem) along with an expert in research methods (José Galván). Moreover, some common errors when writing an article are presented; at last, an integration of the different text composition stages with some self regulation strategies is proposed, as a possible guide to orientate the strategic activity used by the authors when approaching the process of writing a review article in Education and Psychology.
\end{abstract}

Key Words: Review Papers, APA, research methodology.

1 Correspondencia a: 1) Carlos E. Zerpa: Ciudad Universitaria de Caracas, Edificio del Decanato de Ingeniería, piso 3, Departamento de Educación para Ingeniería. Los Chaguaramos, Caracas. Email: carlos.zerpa@ucv.ve 2) Jorge J. Ramírez L. Universidad Simón Bolívar, Edificio de Estudios Generales, Departamento de Ciencia y Tecnología del Comportamiento, primer piso. Sartenejas, Baruta, Edo. Miranda, Venezuela. Apartado postal 89000 Email: jjramirez@usb.ve Teléfonos: 58212-605-3084 (Ofic.-UCV); 58-414-126-8518 (Cel.)./58-212-906-3810/3811/3815 (Ofic-USB.); 58-414-365-0995. 


\section{Introducción}

Frecuentemente, las tareas que el cuerpo docente de un programa de postgrado asigna a sus estudiantes suelen ser motivo de preocupación e inclusive de deserción al constituirse como exigencias problemáticas y de elevada dificultad. No son pocos los casos en que las personas inscritas en un curso desarrollan aversión a la realización de trabajos escritos pero posiblemente esto ocurre más por un bajo desarrollo de la habilidad de componer textos o la carencia de estrategias y directrices claras para su realización que a deficiencias de otro orden.

En tal sentido, en este trabajo se presentan, de forma breve, algunas ideas relevantes para la elaboración de artículos de revisión, una clase particular de investigación que es frecuente encontrar en las principales revistas especializadas en los campos de la Educación, la Psicología y ciencias sociales en general.

\section{Las Diferentes Intenciones de Comunicación de los Artículos de Investigación}

Desde una perspectiva general, puede decirse que cuando se escribe un artículo para una revista científica o especializada es necesario partir de una intención de comunicación específica. En otras palabras, el punto de partida gira alrededor de un par de preguntas: ¿qué se quiere decir? y ¿cómo se puede decir? Algunos modelos de procesamiento de textos, especialmente el de Kintsch y van Dijk (1978) han propuesto una organización macroproposicional que genera estructuras específicas de textos expositivos. En todo caso, la idea que quiere explicarse aquí es la posibilidad de categorizar o clasificar las publicaciones de revistas en diferentes tipos, pues no todos los propósitos de comunicación de los autores son iguales.

El Manual de Estilo de Publicaciones de la American Psychological Association (APA), en su edición en español de 2002, caracteriza varios tipos de artículos de investigación usualmente publicados en revistas especializadas. En tal sentido, existen al menos cinco (5) categorías, como se puede observar en la Tabla 1:

\begin{tabular}{|c|c|c|c|c|c|}
\hline \multicolumn{6}{|c|}{ Tipos de Articulos de Investigación } \\
\hline & $\begin{array}{c}\text { Informe estudios } \\
\text { empiricos }\end{array}$ & Anticulo de Rerisión & Anticulo Teórico & $\begin{array}{c}\text { Artículo } \\
\text { Metodológico }\end{array}$ & Otros \\
\hline 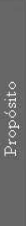 & $\begin{array}{l}\text { Reporte de investigación } \\
\text {, busca dar a conocer } \\
\text { resultados empiricos } \\
\text { especificos en el que se } \\
\text { emplean métodos de } \\
\text { corte experimental, } \\
\text { cuasi experimental, } \\
\text { estudio de campo, otros. }\end{array}$ & $\begin{array}{l}\text { Documento en el que se } \\
\text { hacen criticas o } \\
\text { comentarios sobre } \\
\text { articulos publicados. } \\
\text { Presenta avances o } \\
\text { estado del arte del tema } \\
\text { Puede incluir meta- } \\
\text { análisis, evalua el tema } \\
\text { conforme a la literatura } \\
\text { existente. }\end{array}$ & $\begin{array}{l}\text { Se proponen av ances de } \\
\text { las teorias a partir de la } \\
\text { literatura disponible. } \\
\text { Intenta precisar, } \\
\text { expandir o criticar punto } \\
\text { de vista, busca mostrar } \\
\text { imprecisiones, } \\
\text { ambiguedad } \\
\text { contradicciones. }\end{array}$ & $\begin{array}{l}\text { Exponen alternativas de } \\
\text { métodos, } \\
\text { modificaciones o nuevas } \\
\text { aproximaciones en el } \\
\text { abordaje de un problema } \\
\text { de investigación. }\end{array}$ & $\begin{array}{l}\text { Estudios de casos. } \\
\text { Informes breves. } \\
\text { Comentarios y/0 } \\
\text { réplicas a estudios } \\
\text { previos. } \\
\text { Discusión métodos } \\
\text { cuantitativos. } \\
\text { Historia de casos. } \\
\text { Monografias. }\end{array}$ \\
\hline 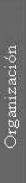 & \begin{tabular}{|l} 
- Introducción \\
- El Método \\
- Resultados \\
- Conclusiones
\end{tabular} & $\begin{array}{l}\text { - Definición del } \\
\text { Problema } \\
\text { - Sintesis de } \\
\text { Investigación. } \\
\text { - Identifica relaciones } \\
\text { contradicciones, vacio } \\
\text { de información, } \\
\text { inconsistencias. }\end{array}$ & $\begin{array}{l}\text {-Similar a un artículo de } \\
\text { revisión, pero pueden } \\
\text { presentar información o } \\
\text { datos empincos. }\end{array}$ & $\begin{array}{l}\text { Sugieren sep arar en } \\
\text { apéndices la } \\
\text { información especifica } \\
\text { del método, pruebas, } \\
\text { cáculos. }\end{array}$ & - Según cada caso. \\
\hline
\end{tabular}

Tabla 1.Tipo de Artículos de Investigación - Manual de Estilo de Publicaciones APA, 2002.

Informes de estudios empíricos. En ellos se reportan investigaciones originales en las que se emplea un método de corte experimental, pre-experimental, estudio de campo u otro y tiene como propósito dar a conocer los hallazgos empíricos (datos) y el resultado de los análisis estadísticos o de otro tipo que se hayan efectuado a los datos recolectados durante el procedimiento.

Usualmente, este tipo de estudio se estructura en cuatro apartados: 1) la introducción, en la que se desarrolla el asunto-problema del artículo y se formulan los objetivos del trabajo; 2) el método; 3) los resultados, donde se destacan los hallazgos del análisis de los datos y se presentan tablas descriptivas de los estadísticos empleados; 4) la discusión, en la que se explican los resultados a la 
luz de los aspectos conceptuales seleccionados para organizar el marco referencial, se interpretan los hallazgos y se concluye en torno a los resultados obtenidos.

Artículos de revisión (de reseña o recensión). Se trata de documentos en los que se hace una crítica o se hacen comentarios en torno a artículos que ya han sido publicados. Presenta los avances o el estado del arte del tema seleccionado; o bien, hace una exploración de los hallazgos sobre la temática en un periodo de tiempo determinado. Puede incluir a los estudios realizados a través de meta-análisis; en términos generales en estos artículos se hace evaluación de un tema dado conforme a la literatura existente hasta la fecha en que se realizaron. El Manual de Estilo de Publicaciones APA (2002) sugiere que en este tipo de artículo: 1) se define y clarifica el problema (o el tema en cuestión); 2) se sintetiza el conjunto de investigaciones preliminares sobre la temática, con el propósito de exponer sobre los avances o el curso de la investigación en el área; 3) puede identificar relaciones, contradicciones, vacíos de información o explicación e inconsistencias en la literatura sobre el tema; 4) puede proponerse el análisis operacional requerido para resolver las dificultades o problemas que surgen a partir de la revisión (o lo que se indica en 1, 2 y 3). Los componentes de estos artículos se organizan en términos de relaciones más que en términos de una estructura de reporte experimental típico de los estudios empíricos.

Artículos teóricos. Se trata de textos, documentos, en los que se proponen avances conceptuales sobre un tema particular a partir de la investigación existente; en otras palabras, este tipo de artículo contribuye al avance de las teorías. De acuerdo al Manual de Estilo de Publicaciones APA (2002) estos artículos pueden tener una estructura similar a los artículos de revisión pero se diferencian en que estos últimos pueden presentar información o datos empíricos cuando las elaboraciones del autor o la autora afectan o influyen sobre aspectos conceptuales o teóricos.

Los artículos teóricos se caracterizan porque en ellos el autor o la autora intenta precisar, expandir o criticar (incluso refutar) puntos de vista o teorías existentes partiendo del análisis de las mismas y mostrando sus imprecisiones, ambigüedades y contradicciones. Comúnmente, este ejercicio lleva al autor o la autora a plantear una modificación, extensión o corrección de las teorías previas, o bien, al planteamiento de una nueva teoría (descriptiva, explicativa o sustantiva). El examen de la consistencia interna de las teorías existentes suele corresponderse con el trabajo que realiza el autor o la autora; asi mismo, la consistencia externa puede ser su objeto de investigación. En la elaboración de estos documentos, el aspecto de relaciones más que el cronológico, es el foco de atención del autor o la autora.

Artículos metodológicos. Estos documentos exponen alternativas de método, modificaciones en los métodos o nuevas aproximaciones metodológicas en el abordaje de un problema de investigación conocido, en cuanto al análisis cuantitativo de datos. Suelen contener detalles precisos de las maneras de abordaje del problema de investigación del que traten y, en especial, deben permitir la posibilidad de hacer las comparaciones pertinentes entre los métodos empleados en las investigaciones preliminares sobre el tema con las alternativas, ampliaciones o propuestas novedosas del autor o la autora. El Manual de Estilo de Publicaciones de la APA (2002) sugiere incorporar en apéndices la información relativa a los detalles de las pruebas, simulaciones, cálculos, entre otros, a fin de facilitar la exposición y comprensión del tema.

Otros artículos. No tan frecuentes ni muy citados son los estudios de casos, informes breves, comentarios y réplicas a estudios publicados previamente, discusiones de métodos cuantitativos, historias de casos y monografías. Básicamente, se espera que tales documentos (al igual que los anteriores) tengan una estructura lógica que les confiera consistencia interna o coherencia lineal, de acuerdo al modelo de tratamiento de textos de Kintsch y van Dijk (1978).

\section{Sugerencias de Daryl Bem (1995).}

Se mencionó anteriormente la naturaleza y la estructura y organización que convenientemente debe tener un artículo de revisión. Bem (1995) ha propuesto algunas ideas de importancia (o más bien sugerencias) que pueden contribuir en la tarea de escribir este tipo de artículos. 
Antes de la elaboración del documento.

Antes de redactar, resulta importante tener claro que la intención de escribir sobre un tema o problema dado resulte de interés o sea significativo para la audiencia a la que irá dirigido. Si es un público especializado en el tema se supone que el aporte debe ser tal que aborde en profundidad lo que se estudia. No es lo mismo un público lector de un área que de otra (lo que sugiere la necesidad de evaluar el tipo de publicación al que se enviará el artículo; esto incluye conocer si la línea editorial se ajusta al tipo de documento que se pretende elaborar). Si la contribución no aporta nada nuevo al área temática o la comunidad especializada en el tema, sencillamente el esfuerzo puede resultar frustrante. Resulta imprescindible garantizar la calidad de la propuesta antes de iniciar el esfuerzo a realizar, y considerar que un buen escritor o una buena escritora debe hacer las veces de un/a buen/a docente.

Durante el proceso de redacción.

Los dos criterios clave para la realización del escrito son, de acuerdo con Bem (1995) la exactitud y la claridad. Con respecto a la claridad, lo que se sugiere es escribir de forma simple y directa. Se trata de utilizar un lenguaje llano, no cargado de palabras sobre-elaboradas o bien, se debe cuidar de no omitir los aspectos del lenguaje que resulten imprescindibles para obtener un documento con apropiada redacción y estilo.

Bem sugiere atender a los aspectos siguientes para cubrir los dos criterios ideales en la redacción de un artículo de revisión, como se observa en la Figura 1.

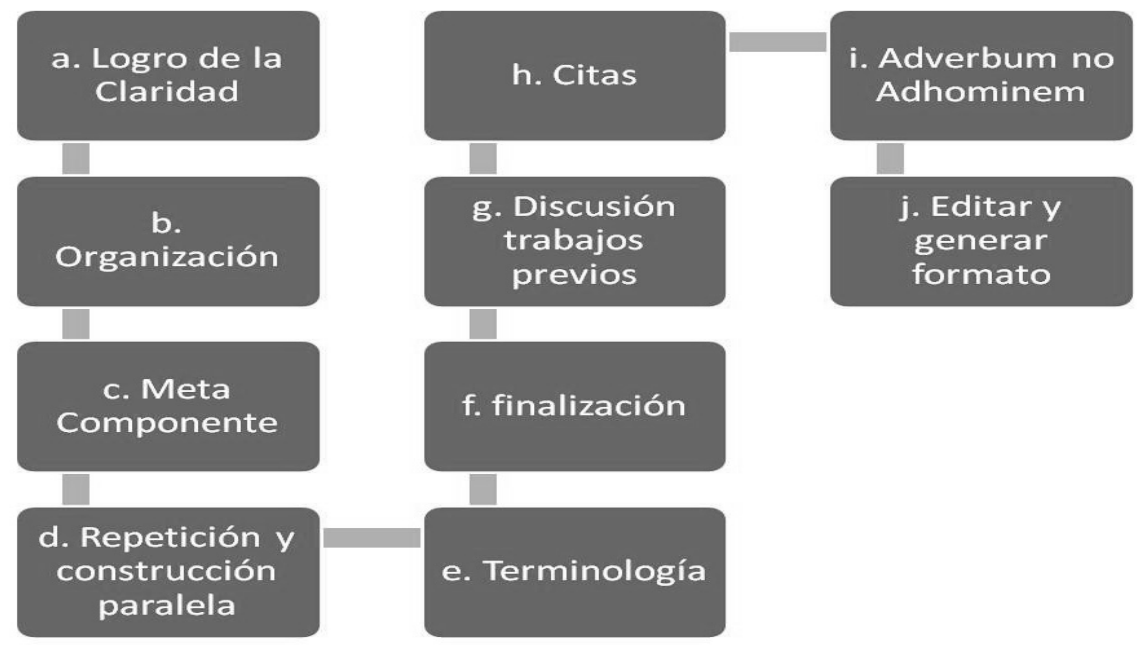

Figura 1. Sugerencias para escribir un artículo de revisión (Bem, 1995)

a) El logro de la claridad: se debe procurar escribir de forma simple y directa. Seguir el famoso dictamen de "omitir palabras innecesarias" y por extensión conceptos innecesarios, tópicos, anécdotas, aspectos colaterales, y notas de pie de páginas. Si un tópico es tangencial para los argumentos centrales, es necesario proceder a eliminarlo.

b) Organización: como segundo paso para la claridad, se debe redactar el texto de forma coherente; un artículo de revisión resulta más difícil de organizar que un reporte empírico. Una manera conveniente de organización puede ser, por ejemplo, destacar al inicio las conclusiones comúnmente aceptadas sobre el tema; luego agregar datos de apoyo o datos que se opongan a las mismas, presentados en un orden descendente de "nivel probatorio" (referido a la fuerza que puedan tener para generar conclusiones convincentes) y contra-argumentar para destacar lo desconocido o lo que no está muy claro en la literatura. Esto puede convencer a lectores/as escéptico/as. Finalmente, se pueden resumir las razones o argumentos que se esgrimen como 
autor/a para generar las conclusiones.

c) Metacomentarios: puede ayudar a dar una visión rápida de la estructura y contenido del documento, pero debe evitarse hacer metacomentarios acerca de la escritura. La exposición puede fallar si distrae la atención del lector en aspectos distintos al tópico central del trabajo; el proceso de escritura debe ser invisible para el lector. La prosa propia debe dirigir el flujo de la narración, sin necesidad de fungir como guía turística; por ejemplo: en vez de decir “...ahora que las tres teorías de la emoción han sido descritas, podemos comenzar con el estudio de los trabajos empíricos de cada una de ellas.”, diríjase directamente a la discusión de la teoría de interés con una simple transacción en la oración como la siguiente “...cada una de las tres teorías han sido probadas empíricamente. Por lo tanto, la teoría del modelo multialmacén ha recibido apoyo en la realización de estudios que...”. La necesidad de guía puede ser suplida por el uso de encabezados o títulos y por la repetición y construcción paralela.

d) Repetición y construcción paralela: Los escritores o las escritoras con poca experticia a menudo sustituyen sinónimos para recurrir a otras palabras y variar la estructura de las oraciones bajo la creencia errónea de que el uso de otras palabras es más creativo e interesante. Es preferible utilizar la repetición y la construcción paralela. Por ejemplo: "las mujeres pueden ser más expresivas que los hombres en el dominio de emociones positivas, pero ellas no son más expresivas en el dominio de emociones negativas". No resulta creativo decir "las mujeres son más expresivas que los hombres en el dominio de emociones positivas pero no es el mismo caso el hecho de que ellas son más propensas que el sexo opuesto para exhibir menos afectos de alegría”. En este caso, la creatividad puede ser interesante pero definitivamente confusa. En la escritura científica suele ser erróneo cuando un autor utiliza diferentes palabras o sinónimos para referirse a un mismo concepto.

e) Terminología. El empleo de la terminología especializada en una disciplina (lo que se suele llamar "jerga”) sirve para legitimar las funciones de la comunicación científica. Un término especializado puede ser más general, preciso y libre de significado excesivo que su equivalente en lenguaje natural.

f) Finalización. Muchos artículos terminan convenientemente con el planteamiento de interrogantes que no han sido respondidas a lo largo del texto; se suelen plantear sugerencias para que futuras investigaciones puedan ayudar a resolverlas. El sugerir aspectos a ser investigados en futuros trabajos resulta ser la vía más común de finalizar un artículo.

g) Discutir trabajos previos, sus métodos y resultados. Puede hacerse una descripción de la variación de procedimientos (empleados en las investigaciones preliminares) en un orden cronológico, permitiendo esto una visión general de tales estudios de forma muy conveniente. El resumir los trabajos de forma clara y precisa suele ser un trabajo que implica varias lecturas y re-escrituras.

h) Citas. Los artículos de revisión (por ser de re-visión) suelen incluir un número mayor de referencias y citas que otro tipo de artículo.

i) Ad verbum, no Ad hominem. Luego de revisar las investigaciones anteriores es tarea común hacer libre crítica, de forma comedida, tanto como se considere necesario, en consonancia con los aspectos que se evidencia como contradictorios, deficientes o se consideran inapropiados. Pero debe criticarse el trabajo y los argumentos, mas no en lo personal al investigador o investigadora, autores/as, revista o revisores del artículo. Dirigir las críticas a los argumentos, y no a la persona resulta una práctica que favorece el desarrollo de la ciencia.

j) Editar y dar formato: Una vez que se ha hecho la revisión y el ajuste de la edición del texto, las referencias, el uso de tablas y figuras, la numeración de páginas, entre otros elementos del manuscrito al formato APA (o el que se esté empleando, normas UPEL 2006 o guías metodológicas como la de Pérez (2004), por ejemplo), es necesario asegurarse de considerar las normas específicas de cada editor o revista a la que enviará el artículo.

Después de la elaboración del manuscrito, se debe considerar:

Luego de atender todos los puntos anteriores, es prudente tomarse un tiempo para revisar la redacción y considerar re-escribir algunos tópicos. Suele ser difícil revisar la propia escritura y 
detectar problemas o brechas de información ya que el autor o la autora conoce lo que se quiere decir. Una práctica es dejar el manuscrito por un tiempo, y retomarlo cuando se esté menos familiarizado. Al leerlo, puede intentarse realizar preguntas como: ¿ya se definió este concepto?, ¿está soportada con referencias o investigaciones una determinada afirmación?, ¿¿se puede precisar en determinado estudio cuáles fueron las variables utilizadas?

También suele ser una buena estrategia entregar el manuscrito a colegas para una revisión crítica, asegurándose de no asumir posturas defensivas, ni argumentativas ante las críticas; frente a un comentario de falta de claridad, lo mejor es aceptarlo; luego se debe procurar precisarlo y revisarlo posteriormente. La práctica de agradecer las observaciones es ética y conveniente.

Re-escribir: Es práctica común recibir observaciones de los árbitros de revistas; cuando se tienen a mano, hay que prestar especial atención y cuidado a los comentarios y no rendirse ante el esfuerzo por muy exigentes que estos sean; resulta muy conveniente consultar con colegas y expertos los requerimientos; otra estrategia suele ser pensar que el documento es una versión piloto o inicial que aún está en proceso de elaboración y que las críticas pueden ayudar a mejorarlo.

\section{Sugerencias básicas de José Galván (1999).}

Galván (1999) presenta un esquema de tres grandes fases en el proceso de elaboración de artículos de revisión: 1) seleccionar un tópico de un campo que pueda ser nuevo para el investigador o la investigadora; 2) identificar y localizar un apropiado número de artículos utilizando bases de datos y revistas especializadas; 3 ) escribir y editar un ensayo bien desarrollado en un tiempo prudencial. Normalmente, este tipo de documento puede realizarse sin mucha supervisión especializada (un tutor o tutora, un/a docente, por ejemplo), pero se espera que el autor o la autora genere un documento con suficiente profundidad en su contenido y escrito correctamente.

\section{Sugerencias para escribir artículo de revisión(Galván, 1999):}

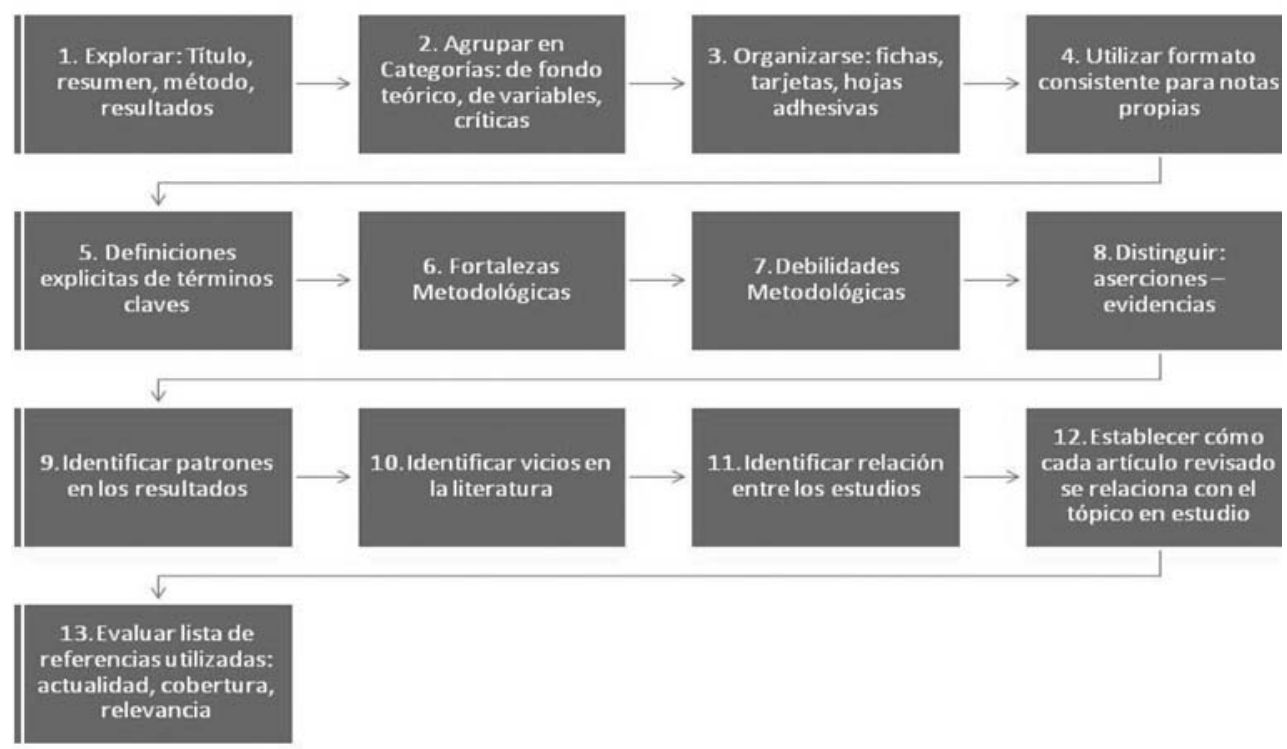

Figura 2. Sugerencias para escribir un artículo de revisión (Galván, 1999) 
Puntualmente, Galván sugiere los siguientes aspectos (ver Figura 2):

1) Explorar los artículos obtenidos y hacer una revisión general de cada uno de ellos; es decir, hay que leer atendiendo principalmente a lo siguiente: título, resumen, método (participantes, medidas), resultados y discusión. 2) Sobre la base de la revisión de los artículos (conforme al tema o problema seleccionado), agruparlos por categorías. Por ejemplo: a) revisiones de fondo (fondo histórico general; bases filosóficas; aspectos legales); b) efectos de la variable en cuestión (sobre el rendimiento académico, sobre un grupo minoritario, etc.); c) críticas; d) alternativas para las afirmaciones expuestas. 3) Organizarse antes de leer los artículos (se requieren por ejemplo, equipos, tarjetas para hacer fichas y escribir comentarios, hojas adhesivas, etc.).

4) Utilizar un formato consistente para las notas propias (como investigador o investigadora). Una vez que se han organizado los artículos hay que leerlos destacando puntos de importancia y escribir notas en fichas o tarjetas. Por ejemplo: a) autor; título; año de publicación; nombre de la publicación; b) ¿cuál es el tema principal o punto fundamental del artículo?; c) describir la metodología empleada; d) describir los hallazgos; e) ¿qué es lo más resaltante del artículo?; ¿es un estudio experimental?; ¿es un estudio cuantitativo o cualitativo? El uso de estrategias de organización de la información puede ser muy útil en esta fase (esquemas, mapas mentales o conceptuales); f) anotar otros aspectos destacables del artículo.

5) Buscar definiciones explícitas de términos clave en la literatura. 6) Buscar fortalezas metodológicas.7) Buscar debilidades metodológicas. 8) Distinguir entre aserciones y evidencias. 9) Identificar las mayores tendencias o patrones en los resultados de estudios previos. 10) Identificar vacíos en la literatura. 11) Identificar relaciones entre los estudios. 12) Establecer cómo cada artículo revisado se relaciona con el tópico elegido para la investigación. 13) Evaluar la lista de referencias bibliográficas utilizada (los artículos empleados para elaborar el documento) con base en su actualización (se suele pensar que la literatura debe ser reciente, aunque puede ser relativo, dependiendo del tema) y cobertura (o profundidad/calidad sobre el tema).

Para el análisis de la literatura desde el punto de vista del investigador o la investigadora, Galván sugiere considerar ciertos aspectos al abordar cada artículo seleccionado, como se observa en la Figura 3.

\begin{tabular}{l}
\multicolumn{1}{c}{$\begin{array}{c}\text { A considerar para cada } \\
\text { Artículo }\end{array}$} \\
- 1. Precisar tipo de investigación: \\
cuantitativa o cualitativa. \\
- 2. Identificar: estudio experimental \\
o no experimental. \\
- 3. Si es experimento: verificar \\
asignación de participantes a \\
condiciones experimentales y de \\
control. \\
4. Examinar los indicios de las \\
relaciones de causa-efecto en no \\
experimentales. \\
- 5. Identificar las variables \\
estudiadas. \\
6. Examinar las caracteristicas de la \\
muestra. \\
7. Verificar la existencia de \\
diferencias estadisticamente \\
significativas.
\end{tabular}

Para sintetizar literatura elegida

- 1. Considerar el propósito antes de empezar a escribir.

- 2. Considerar cómo relacionar las notas realizadas.

- 3. Crear un tópico base sobre el que se sustente un argumento central para la discusión.

- 4. Reorganizar las notas conforme al argumento.

- 5. Para cada subtítulo, precisar las relaciones entre estudios, buscar vacios de información o necesidad de más investigación.

- 6. Planear-discutir cómo los estudios se relacionan y avanzan en nociones teóricas.

- 7. Planear-presentar conclusiones e implicaciones y sugerir directrices para futuras investigaciones.

Figura 3. Aspectos a considerar para cada artículo revisado y sugerencias para la síntesis de la literatura elegida (Galván, 1999). 
Otro aspecto de gran relevancia que puede ser de interés para los/las autores/as interesados/as en escribir un artículo de revisión, es conocer algunos de los errores más comunes frecuentemente reportados por jueces y evaluadores de revistas científicas. Al respecto, Baumeister (2003) presenta una síntesis de los principales errores, a saber (ver Figura 4):

1) Propósitos poco claros o inciertos. Algunos pueden pensar que el objetivo es hacer un resumen de los trabajos pasados, pero un simple resumen por lo general no logra hacer una contribución que sea fácilmente publicable.

2) Introducción vaga, pobre organización: la introducción debe especificar el objetivo de la revisión, además de explicar cuidadosamente la teoría; la organización del documento e ideas resultan un aspecto crucial a considerar.

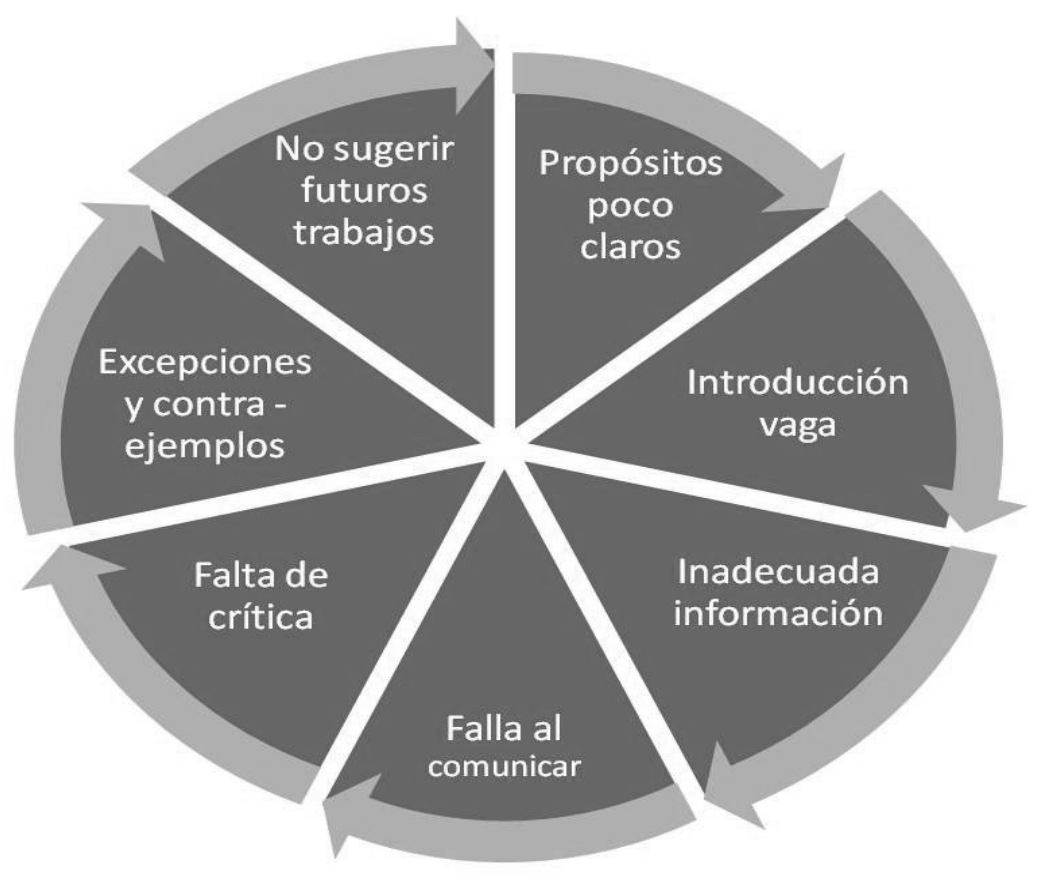

Figura 4. Errores comunes en un artículo de revisión de la literatura (Baumeister, 2003)

3) Inadecuada información: poca información y precisión sobre la literatura revisada; en otros casos puede suceder lo contrario, exceso de detalles e información sobre los estudios revisados. Falta de relación entre la información.

4) “Take - Home message”. Falla al comunicar el mensaje; está relacionado con el propósito del artículo, más que sólo el resumen de la teoría.

5) Falta de crítica. Un error es no fijar posiciones críticas frente a aspectos de la teoría que se revisan. En Educación, Psicología y otras áreas de las ciencias sociales, las teorías no suelen estar libre de críticas; una forma de realizarlo es agrupar un conjunto de investigaciones que compartan alguna teoría y luego formular alguna crítica al respecto. Olvidar hacer referencia a aspectos críticos es un error común que se comete frente a la teoría asumida.

6) Excepciones y contraejemplos. Resulta un error común la falta de análisis de excepciones; se sugiere colocar hacia el final del documento una pequeña sección de evidencias contradictorias lo cual tiene como ventajas ayudar a buscar evidencias que permiten monitorear y ajustar las propias conclusiones; esta práctica también ayuda en la precisión de comentarios, dado que puede ser más convincente para el lector o la lectora el mostrar amplitud en el análisis; este apartado puede ayudar a desarrollar una interesante y posiblemente compleja formulación a la teoría.

7) No decir hacia dónde se orienta la investigación futura. Dejar de proporcionar información crítica referida a las posibles vías que pueden tomar las futuras investigaciones, suele ser otro error común. 
Por último, siguiendo a Baumeister (2003), uno de los errores más comunes se relaciona con estilos de presentación inadecuados. La revisión de la literatura es más efectiva al asumir algún estilo de presentación y discusión como el manual de estilos APA (2002) (se mencionó antes también el estilo UPEL, 2006). Resulta necesario aclarar en el manuscrito aspectos tales como distinguir cuando un autor o autora refiere alguna opinión, especulación o comentario, y cuando utiliza pruebas o muestra como producto un resultado, una opinión y/o evidencias empíricas. En la revisión se debe cubrir ambos aspectos, pero se debe dejar claro al público meta cuál es el aspecto tratado en cada momento.

Elementos para la auto-regulación del proceso de composición de un artículo de revisión.

Además de los elementos de índole formal planteados, escribir un artículo de revisión implica el desarrollo de ciertas habilidades personales, cognitivas y conductuales necesarias para asumir dicha actividad, diversos autores (Zimmermann y Risemberg, 1997, 1998; Díaz y Hernández, 2002), han propuesto aproximaciones considerando aspectos de la auto-regulación que proporcionan luces y pueden traducirse en una guía para la acción.

Díaz y Hernández (2002) refieren un modelo general de composición de textos en el cual un escrito puede organizarse de acuerdo a dos grandes estrategias: la primera vinculada al aspecto estructural del documento y la segunda referida al aspecto funcional del escrito. En relación con la primera de las estrategias, la composición atiende a una estructura de tres partes o fases en la composición del texto: una fase de planificación, una fase de textualización y una fase de evaluación. En la planificación se requiere que el autor o la autora del manuscrito desarrolle un plan de texto o prototipo de cómo visualiza el artículo concluido, clarificando el propósito, los apartados que lo conformarán, los recursos gráficos que lo apoyarán y otros aspectos que orienten la redacción del texto hacia una dirección seleccionada por el autor (la elaboración de un diagrama o mapa mental suele ser una buena herramienta para organizar lo planificado).

En la fase de textualización, se espera que el autor utilice una serie de recursos para el monitoreo o supervisión de la redacción del escrito, a fin de verificar que cada sección se ajusta a la idea especificada en la fase de planificación. La supervisión de la coherencia lineal del texto, la sintaxis, la transcripción y la revisión semántica de lo que se dice, es una tarea de máxima importancia en esta etapa. Si el monitoreo sugiere que hay diferencia entre lo que se está componiendo y el plan original resultará necesario ir atrás y re-iniciar el trabajo de escritura hasta lograr el mejor ajuste. Por último, en la fase de evaluación se procura hacer una retrospectiva y revisar si el documento finalizado se corresponde con el documento planificado.

En relación a una dimensión funcional, los autores refieren a aspectos considerados a la planificación estructural y sugieren formularse y responder las siguientes interrogantes:

-¿Qué se quiere decir?: se refiere a la habilidad para establecer claramente cuál es el tema central del texto y lo que se desea explicar, describir, analizar o evaluar sobre dicho tema. Gall, Borg y Gall (1996) aportan cinco criterios que condicionan este aspecto: a) delimitar el problema de investigación; b) verificar aproximaciones fructíferas en el tema; c) obtener elementos metodológicos claves de las investigaciones o fuentes primarias y secundarias revisadas; d) identificar recomendaciones para investigaciones futuras; y e) buscar apoyo para teorías con base en lo que se investigue.

-¿Cómo se puede decir?: vinculado con la destreza discursiva en estilo y redacción del manuscrito, uso de pistas tipográficas y discursivas para destacar los aspectos más relevantes, organización de las ideas en estructuras textuales y revisión continua de la exposición de las ideas que se quieren incluir.

-¿Para qué quiere decirse?: referido a la necesidad de verificar que en el desarrollo del documento exista coherencia entre lo que se propone como objetivo del manuscrito y lo que finalmente resulta; igualmente, Bem (1995) y Galván (1999) aportan acciones concretas para realizar el monitoreo de este aspecto. 
-¿Para quién quiere decirse?: vinculado con el público meta al cual va dirigido el documento. Es necesario incluso evaluar preliminarmente la línea editorial de la revista a la que se piense enviar el artículo de revisión.

Más allá de lo anterior, y en otro orden de ideas, el modelo de auto-regulación del aprendizaje de Zimmermann y Risemberg (1997) constituye otra referencia útil en la actividad de elaboración de un artículo de revisión. Este modelo considera que los procesos ejecutivos de una actividad involucran cuatro aspectos interrelacionados de vital importancia para el logro eficiente de actividades complejas como la composición de textos, a saber: la auto-observación-evaluación, el establecimiento de metas u objetivos, la implementación y monitoreo de estrategias y la evaluación de los resultados. Tales aspectos pueden incluir diversos procesos auto-regulatorios que dirigen el proceso de la realización de la tarea, a saber: a) ajuste de metas, b) estrategias de la tarea, c) visualización, d) auto-instrucción, e) manejo del tiempo, f) auto-monitoreo, g) auto-evaluación, h) auto-consecuencias, i) estructuración del ambiente, j) búsqueda de ayuda.

Finalmente, el ensayar una integración de los modelos de fases de composición de texto y de estrategias de auto-regulación en el proceso de escritura de un artículo de revisión, a pesar de pertenecer a áreas cognitivas vinculadas pero con diferentes direcciones en su concepción, se estima un marco que pudiese ser orientador y de apoyo durante todo el proceso de composición del texto, especialmente por autores con moderada experiencia.

En la tabla 2 se presentan algunos ejemplos de cómo para cada una de las tres fases de composición de texto, se pueden aplicar estrategias de auto-regulación; sin embargo cada autor/a debe adaptar las estrategias a sus características individuales y requerimientos particulares que lo impulsan a escribir un artículo de revisión en Educación y/o Psicología.

En efecto, la tabla 2 expone una ilustración de la aplicación del modelo de Zimmermann y Risemberg $(1997,1998)$ adaptada de forma sucinta a un ejercicio de elaboración de un artículo de revisión. Como se observa, el conjunto de estrategias identificadas es operacionalizable en acciones concretas que desde un marco auto-regulatorio dirigen las cogniciones, el comportamiento y las actitudes mismas que se involucran en el desarrollo del proceso. Cada estrategia es verificable de esta manera y arrojan en su conjunto una lista de referencia de metas y sub-metas a cumplir. Nótese que tales estrategias se agregan en función del aspecto estructural de la composición del texto, expuestas por Díaz y Hernández (2002), conformando así un marco integrador para el control de la elaboración del documento. 


\begin{tabular}{|c|c|c|c|}
\hline $\begin{array}{l}\text { Estrategia de } \\
\text { Auto-regulación }\end{array}$ & Fase de Planificación & Fase de Textualización & Fase de Evaluación \\
\hline $\begin{array}{l}\text { Estrategias para } \\
\text { la tarea }\end{array}$ & $\begin{array}{l}\text { ¿Qué se quiere decir? } \\
\text { ¿Cómo se puede decir? } \\
\text { ¿Para qué quiere decirse? } \\
\text { ¿Para quién o quiénes? } \\
\text { Evaluar guía propuesta por } \\
\text { Bem (1995) y Galván (1999). }\end{array}$ & $\begin{array}{l}\text { Conocer cómo ejecutar el } \\
\text { proceso de escritura y buscar } \\
\text { mejorar. } \\
\text { Identificar momento y día } \\
\text { de mayor energía, aspectos } \\
\text { motivacionales. } \\
\text { Ver propuesta Bem (1995) }\end{array}$ & $\begin{array}{l}\text { Identificar estrategias } \\
\text { utilizadas y evaluar su } \\
\text { efectividad en función } \\
\text { de resultado de artículo. } \\
\text { Considerar ajustes. } \\
\text { Verificar lista errores } \\
\text { comunes Baumeister (2003). }\end{array}$ \\
\hline Ajuste de metas & $\begin{array}{l}\text { Definir propósito, esquema } \\
\text { del artículo, potenciales } \\
\text { logros y tiempo estimado en } \\
\text { horas para cada fase. }\end{array}$ & $\begin{array}{l}\text { Establecer metas } \\
\text { Crear listas de avances } \\
\text { diarios. } \\
\text { Fijar metas diarias o } \\
\text { semanales de escritura. }\end{array}$ & $\begin{array}{l}\text { Verificar pertinencia del } \\
\text { manuscrito y relación entre lo } \\
\text { planificado y lo ejecutado. }\end{array}$ \\
\hline $\begin{array}{l}\text { Visualización } \\
\text { o empleo de } \\
\text { imágenes para } \\
\text { anticiparse } \\
\text { al producto } \\
\text { terminado de } \\
\text { forma exitosa }\end{array}$ & $\begin{array}{l}\text { Imaginar el artículo listo para } \\
\text { ser arbitrado. } \\
\text { Visualizar consecuencias } \\
\text { asociadas a la publicación del } \\
\text { texto, reconocimiento. }\end{array}$ & $\begin{array}{l}\text { Visualizar que cada día } \\
\text { mejora su ejecución, claridad } \\
\text { en plantear ideas, capacidad } \\
\text { de síntesis e integración, } \\
\text { facilidad de expresión. }\end{array}$ & $\begin{array}{l}\text { Visualizar cómo posibles } \\
\text { comentarios de árbitros o } \\
\text { evaluadores le ayudarán } \\
\text { a continuar mejorando la } \\
\text { calidad de su artículo. }\end{array}$ \\
\hline Auto-instrucción & $\begin{array}{l}\text { Verbalizar freses como: } \\
\text { "Quiero comenzar a planificar } \\
\text { mi artículo" } \\
\text { "En x días tendré listo un } \\
\text { primer borrador del artículo” }\end{array}$ & $\begin{array}{l}\text { “A pesar de lo complejo } \\
\text { de escribir, sé que con } \\
\text { dedicación y esfuerzo lo } \\
\text { puedo lograr”. } \\
\text { "Encontraré la forma de } \\
\text { superar los obstáculos que se } \\
\text { me presenten cada día”. }\end{array}$ & $\begin{array}{l}\text { "Cada momento desarrollo } \\
\text { mi habilidad para escribir y } \\
\text { detectar aspectos a mejorar en } \\
\text { mis escritos". }\end{array}$ \\
\hline $\begin{array}{l}\text { Manejo del } \\
\text { tiempo }\end{array}$ & $\begin{array}{l}\text { Establecer un horario semanal } \\
\text { de los dís y horas que } \\
\text { dedicará al artículo. } \\
\text { Dividir las actividades } \\
\text { complejas (objetivos) } \\
\text { en metas cortas, asignar } \\
\text { un tiempo y convertir en } \\
\text { pequeños triunfos. }\end{array}$ & $\begin{array}{l}\text { Monitoree si el logro de cada } \\
\text { meta ocurre en los plazos } \\
\text { temporales estimados. } \\
\text { Establecer espacios para } \\
\text { revisión diaria o semanal de } \\
\text { los avances. }\end{array}$ & $\begin{array}{l}\text { Evaluar si el tiempo dedicado } \\
\text { a cada objetivo / artículo } \\
\text { se corresponde con el } \\
\text { originalmente planificado. }\end{array}$ \\
\hline Auto-monitoreo & $\begin{array}{l}\text { Establecer un sistema de } \\
\text { verificación y registro de los } \\
\text { objetivos identificados para } \\
\text { la tarea. }\end{array}$ & $\begin{array}{l}\text { Definir indicadores de logro } \\
\text { de los objetivos para la tarea. }\end{array}$ & $\begin{array}{l}\text { Cotejar resultados obtenidos } \\
\text { con los resultados esperados } \\
\text { que se identificaron para la } \\
\text { tarea. }\end{array}$ \\
\hline Auto-evaluación & $\begin{array}{l}\text { Evaluar la pertinencia, } \\
\text { relevancia, factibilidad entre } \\
\text { otros aspectos de la propuesta } \\
\text { de artículo. }\end{array}$ & $\begin{array}{l}\text { Evaluar constantemente la } \\
\text { ejecución personal que se da } \\
\text { frente a las exigencias de cada } \\
\text { objetivo. }\end{array}$ & $\begin{array}{l}\text { Juzgar el papel personal } \\
\text { (actitud) asumido durante el } \\
\text { desarrollo de cada objetivo. } \\
\text { Pertinencia, posibles cambios } \\
\text { o refuerzo de aspectos que } \\
\text { facilitaron o dificultaron. }\end{array}$ \\
\hline $\begin{array}{l}\text { Auto- } \\
\text { consecuencias }\end{array}$ & $\begin{array}{l}\text { Identificar agentes } \\
\text { reforzadores de la ejecución } \\
\text { durante la tarea. } \\
\text { Posponer aspectos agradables } \\
\text { hasta terminar de planificar. }\end{array}$ & $\begin{array}{l}\text { Revisar cada pequeño triunfo } \\
\text { y establecer un ritual de } \\
\text { celebración. }\end{array}$ & $\begin{array}{l}\text { Celebrar el logro de las } \\
\text { metas cumplidas y establecer } \\
\text { recompensas para el momento } \\
\text { en que concluya los aspectos } \\
\text { por mejorar. }\end{array}$ \\
\hline $\begin{array}{l}\text { Estructuración } \\
\text { ambiental }\end{array}$ & $\begin{array}{l}\text { Prever las condiciones que } \\
\text { pueden afectar la ejecución: } \\
\text { Lugar, ruido, luz, ergonomía, } \\
\text { materiales necesarios. }\end{array}$ & $\begin{array}{l}\text { Controlar agentes distractores } \\
\text { que obstaculicen el avance } \\
\text { en cada sub-meta: teléfono, } \\
\text { amigos, otros. }\end{array}$ & $\begin{array}{l}\text { Evaluar cómo el ambiente } \\
\text { facilitó o dificultó la } \\
\text { actividad. Identificar aspectos } \\
\text { a reestructurar. }\end{array}$ \\
\hline $\begin{array}{l}\text { Búsqueda de } \\
\text { ayuda }\end{array}$ & $\begin{array}{l}\text { Identificar potenciales } \\
\text { monitores externos para } \\
\text { la co-supervisión de la } \\
\text { tarea: compañeros, amigos, } \\
\text { familiares, pareja. }\end{array}$ & $\begin{array}{l}\text { Buscar sugerencias de } \\
\text { expertos y colegas, plantear } \\
\text { dificultades y buscar } \\
\text { alternativas de solución. } \\
\text { Activar monitores externos } \\
\text { para cada meta y objetivo. }\end{array}$ & $\begin{array}{l}\text { Pedir a expertos, colegas, } \\
\text { amigos sugerencias para } \\
\text { mejorar aspectos del } \\
\text { documento. }\end{array}$ \\
\hline
\end{tabular}

Tabla 2. Ejemplo de fases de composición de texto y estrategias de auto-regulación en el proceso de escritura de un artículo de revisión. 


\section{Conclusiones y recomendaciones}

En este documento se ha pretendido presentar de forma resumida algunas de las ideas esenciales que resultan de importancia considerar cuando un investigador o investigadora se propone escribir un artículo de revisión. Tanto Bem (1995) como Galván (1999), así como Gall, Borg y Gall (1996) refieren aspectos puntuales que se deben atender para que el resultado sea tanto pertinente como de calidad. Así mismo, la propuesta de Baumeister (2003), advierte sobre algunos de los errores frecuentes que presentan los artículos de revisión al ser sometidos a evaluación por jueces o árbitros expertos de revistas científicas.

Por último se presenta una integración de las tres fases estructurales de composición de textos y algunas estrategias de auto-regulación que pudiesen orientar a un/a autor/a o los/las autores/as sobre formas de abordar el complejo proceso de escritura de un artículo de revisión.

Se sugiere no tomar las ideas planteadas a manera de recetario, pues existen tantos propósitos de investigación como investigadores o investigadoras interesados/as en realizar revisiones de literatura en torno a un determinado problema o tema. No obstante, seguir una secuencia organizada de acciones y considerar los aspectos destacados en este documento, pudiera afectar de forma conveniente, una producción escrita consistente, profunda, lógicamente organizada así como una experiencia de crecimiento personal y profesional para los/las autores/as.

\section{Referencias}

American Psychological Association (2002). Manual de Estilo de Publicaciones. México: El Manual Moderno.

Baumeister, R.F. (2003). Writing a Literature review. En M.J. Prinstein y M. Patterson M. The Portable Mentor: Expert Guide to a Successful Career in Psychology. pp. 64-71. New York: Springer.

Bem, D. J. (1995). Writing a review article for Psychological Bulletin. Psychological Buletin, 118(2), 172-177.

Díaz, F., \& Hernández, G. (2002). Estrategias docentes para un aprendizaje significativo. 2da. Ed. México: Trillas.

Gall, M. D.; Borg, W. R. \& Gall, J, P. (1996). Educational Research. An Introduction. New York: Longman Publisher.

Galván, J. L. (1999). Writing Literature Reviews. A Guide for Students of the Social and Behavioral Sciences. Los Angeles: Pyrczak Publishing.

Kintsch, W. \& van Dijk, T. A. (1978). Toward a model of text comprehension and production. Psychological Review, 85, 363-394.

Pérez, A. G. (2004). Guía metodológica para anteproyectos de investigación. Caracas: Fondo Editorial UPEL.

Universidad Pedagógica Experimental Libertador, UPEL (2006). Manual de trabajos de grado de especialización y maestría y tesis doctorales. Caracas: Fondo Editorial UPEL.

Zimmermann, B. J. \& Risemberg, R. (1997). Self-regulatory dimensions of academic learning and motivation. En G. D. Phye (Ed.). Hanbook of academic learning: construction of knowledge. San Diego: Academic Press.

Zimmerman, B. J. \& Risemberg, R. (1998). Academic studying and the development of personal skill: a self-regulatory perspective. Educational Psychologist, 33(2), 73 - 86. 